\title{
EFECTOS POSITIVOS DEL USO DE TECNOLOGÍAS BIOMÉTRICAS PARA EL CONTROL DE ACCESO EN LAS UNIVERSIDADES
}

\author{
${ }^{1}$ Luis Antonio Guevara Aredo Mdol ${ }^{1}{ }^{\mathbf{1}}$ Víctor Antonio Villar García, ${ }^{1}$ Alberto \\ Mendoza de los Santos \\ ${ }^{1}$ Universidad Nacional de Trujillo, La Libertad, Perú
}

Recibido: 05/09/2021 Revisado: 03/10/2021 Aceptado: 16/11/2021 Publicado: 30/01/2022

\section{RESUMEN}

La evolución del uso de los indicadores biométricos para el control de acceso a entornos con equipos e información sensible en conjunto con la actual irrupción de componentes electrónicos y digitales, ha tenido un mayor crecimiento en los últimos años. La presente revisión sistemática abarca el análisis de los efectos positivos que produce el uso de dichos indicadores adheridos a hardware y software, centrando la investigación en el estado actual tanto de universidades a nivel nacional como internacional en técnicas de autenticación, verificación y reconocimiento de usuarios en el campo de la biometría.

En este contexto, responderemos a la siguiente pregunta: ¿Qué efectos positivos conlleva la implementación de tecnologías biométricas para el control de acceso en las universidades? Por lo mismo, se hace necesario centrar como objeto de esta investigación, el impacto que generan las tecnologías biométricas en instituciones de educación superior a partir de la revisión de bases de datos académicas de los últimos 5 años.

Posterior a la revisión sistemática, se logró identificar que los más importantes efectos positivos de las tecnologías biométricas en las universidades son en su mayoría orientadas a la seguridad y fiabilidad tanto de los datos como del entorno, y también, a optimizar el tiempo de desarrollo de los diversos procesos que, a su vez conlleva a un ahorro de tiempo y costos posterior a la aplicación de cualquier sistema con tecnologías biométricas.

PALABRAS ClAVES: Asistencia biométrica, Acceso biométrico, Biometría en Universidades.

\begin{abstract}
The evolution of the use of biometric indicators to control access to environments with sensitive equipment and information, together with the current emergence of electronic
\end{abstract}


and digital components, has had a greater growth in recent years. This systematic review covers the analysis of the positive effects produced by the use of said indicators attached to hardware and software, focusing the research on the current state of both national and international universities in techniques of authentication, verification and recognition of users in the field of biometrics.

In this context, we will answer the following question: What positive effects does the implementation of biometric technologies for access control in universities entail? For this reason, it is necessary to focus as an object of this research, the impact generated by biometric technologies in higher education institutions from the review of academic databases of the last 5 years.

After the systematic review, it was possible to identify that the most important positive effects of biometric technologies in universities are mostly oriented to the security and reliability of both the data and the environment, and also, to optimize the development time of the various processes that, in turn, lead to time and cost savings after the application of any system with biometric technologies.

KEY WORDS: Biometric assistance, Biometric access, Biometrics in Universities.

\section{INTRODUCCIÓN}

Desde una perspectiva general, (Peralta Castellano, 2017) define la biometría como la medición, recopilación y análisis de datos relacionados con las características físicas o de comportamiento de las personas, como medio para verificar su identidad. Tenemos un claro ejemplo de esto cuando hablamos de huellas dactilares o patrones de habla, por lo que es seguro decir que la biometría como técnica de medición refleja características que todos los seres humanos poseen, pero es única en comparación con los demás. Dicho lo anterior, a continuación, presentamos aportes de los investigadores en el Perú y el mundo, respecto a lo obtenido en las universidades, previa implementación de tecnologías biométricas de diversos tipos.

(Bustamante \& Mercedes, s. f., 2017), implementó un sistema de control de registro de asistencia del personal administrativo y docente mediante relojes biométricos y radioenlace en la Universidad Nacional Jorge Basadre Grohmann (Perú Tacna). Los beneficios que se derivan son principalmente el ahorro de recursos económicos, horas de 
trabajo y espacio de trabajo. Además, las firmas biométricas son más fáciles de usar, ya que no necesitan firmar cada vez que entran o salen de las instalaciones.

También, en la Universidad Privada de Pucallpa, (Garcia \& Hidalgo, 2017), implementó un sistema de control de apoyo administrativo biométrico para mejorar la capacidad de control y eliminar sistemas obsoletos y fácilmente manipulables. Esto ha mejorado el desempeño y la productividad del personal administrativo de las escuelas, garantizando la satisfacción de usuarios y seguridad y autenticidad de la información.

(Gastelo, 2019). Diseñó un sistema biométrico de verificación y autenticación de huellas dactilares para la Universidad Tecnológica del Perú (Lima), y su desarrollo condujo a la automatización de los procesos involucrados en el control de la autenticación, participación de docentes y estudiantes.

De la misma forma, (Llanos Malpartida, 2020), implementó un lector biométrico de huellas dactilares para rastrear la asistencia de los estudiantes en la Universidad Nacional Daniel Alcides Carrión (Perú), donde redujo el tiempo que toma registrar la asistencia de los estudiantes, demostrando efectividad y capacidad para dichos procesos.

(Calderon, 2017), estudió el impacto de las tecnologías dactilares en la Fundación Universitaria UNINPAHU (Colombia), donde concluyó que es una alternativa eficaz para controlar la asistencia de personal y estudiantes. Asimismo, verificó que es muy efectiva en el ahorro de tiempo de grabación y costos de material.

(Delia, 2017), presentó la construcción de un sistema de control de acceso para la Universidad Tecnológica Fidel Velázquez (México), la cual se basó en un lector de huella dactilar y un sensor ultrasónico como contador. Logrando que la innovación del sistema fuese una opción para el cuidado de las instalaciones universitarias y generó como mayor éxito, la satisfacción de usuarios.

También, (Duque, 2017), desarrolló un sistema de reconocimiento de huellas dactilares para el control de acceso simulado en un entorno web para la Universidad Libre Sede Bosque Popular (Colombia), demostrando que hace posible sentirse más seguro en un entorno controlable.

Para, (Buriro, 2017), quien desarrolló un sistema de tecnología biométrica facial en la Escuela Internacional de Doctorado en TIC (Italia), obtuvo resultados para minimizar los problemas de inicio de sesión del usuarios en dispositivos móviles, 
basándose en el análisis de los micro-movimientos eliminando problemas de usabilidad y seguridad.

A su vez, en Reino Unido, (Parvinzamir, s. f. 2017), diseñó una aplicación en MATLAB para la verificación de identidad vía huellas dactilares. Donde la ventaja se manifiesta en la presencia del alumno para registrar su presencia, alta aceptabilidad y cobranza, y bajo costo sin hardware y software complejos.

(Belhadj, 2017), para la revista HAL OPEN SIENCE en África del Norte, expone que el sistema de identificación biométrica se ofrece como una alternativa más segura a los sistemas de autenticación tradicionales basados en conocimiento o token. Mejorando mucho su aspecto de seguridad al compensar entre otros métodos en términos de aceptabilidad, precisión, seguridad y tecnología de bajo costo.

(Arteaga Zambrano, 2018), implantó un sistema biométrico para el laboratorio de la Universidad de Manabí (Ecuador), este sistema de huellas dactilares ha permitido la protección completa del área de formación profesional antes mencionada, logrando la seguridad general mediante el acercamiento a la tecnología.

(Silva, 2018), reflejado en un trabajo presentado en el Octavo Simposio de Excelencia en Gestión y Tecnología (Brasil), a raíz del desarrollo de un sistema de control de acceso biométrico de huellas dactilares, demostró que, para garantizar la seguridad en la autenticación de un sistema de control de acceso, se debe adaptar el sistema a hardware que trabaje a la par.

(Anaya Matínez \& Maldonado Arias, 2019), propuso un prototipo de sistema biométrico de huella labial para la identificación y reconocimiento de estudiantes de la Universidad de Santos Tomás (Colombia), que demostró que la liposcopia es una alternativa válida y efectiva para identificar individuos según los patrones impresos en los labios. Estos programas modernos brindan confianza y seguridad en la protección de datos porque están comprimidos en información digital para que puedan protegerse de cambios premeditados y / o cambios accidentales.

(Fernández Villacañas et al., 2019), diseña y fabrica un dispositivo de control de acceso para comedores de universidades de Madrid (España). El objetivo del proyecto es facilitar el acceso de los alumnos y poner a disposición del personal responsable de la supervisión de este espacio datos relevantes.

A su vez, (Tovar et al., 2020), implementó sistemas biométricos faciales para el control de acceso en instituciones de educación superior (entre ellas la Universidad de 
Cartagena en Colombia). La investigación alcanzó un punto importante en la detección de personas con accesorios. Como resultado, un sistema puede reconocer a los usuarios a una distancia de hasta 5 metros.

(Macdonald, 2021) para la revista Biometric Update, comentó que La Universidad Estatal de Agricultura de Stavropol (SSAU) es la primera universidad en el sur de Rusia en implementar la tecnología de reconocimiento facial STC que puede detectar e identificar a los visitantes que ingresan por las puertas de la universidad. El sistema aumenta el nivel de seguridad del campus ya que el acceso a las instalaciones de la universidad estará limitado solo a quienes tengan sus datos almacenados en la plataforma.

(Uzun, 2021), En su investigación para la Universidad de Georgia en Atlanta (EE. UU.), brindó soluciones a los desafíos más sensibles que enfrentan los sistemas biométricos en general (especialmente huellas dactilares), proporcionando a las personas un nuevo protocolo basado en Deep Learning, este enfoque ha llevado a la determinar que el uso del Deep learning para la mejora de tecnologías biométricas, conlleva a volverlo más eficientes, seguros y completos.

En ese sentido, la justificación de la presente investigación se enfoca en dar conocer el impacto positivo que genera las tecnologías biométricas en las universidades, enfocándose en el control de acceso de alumnos, docentes y personal. También, busca brindar conocimiento sobre la seguridad de la información y centralización de datos al analizar todos los efectos positivos. Asimismo, la presente revisión sistemática plantea la pregunta: ¿Cuáles son los efectos positivos de la implementación de tecnologías biométricas en el control de acceso de las universidades a nivel nacional e internacional? Teniendo como objetivo evaluar el impacto que generan dichas tecnologías en el periodo $2017-2021$.

\section{METODOLOGÍA}

Se realizó una revisión sistemática de la bibliografía científica con base en la metodología PRISMA. La pregunta de investigación establecida para conducir el proceso metodológico fue la siguiente: ¿Qué efectos positivos conlleva la implementación de tecnologías biométricas para el control de acceso de las universidades? Así mismo se ha hecho el proceso de búsqueda y recolección de información con algunos términos como lo son: "Biometrics", "Access control and biometrics", "asistencia biométrica", "acceso universitario biométrico", "control de acceso biométrico", "Biometría". Se detalla de 
manera más clara la búsqueda de la literatura científica, haciendo diversas combinaciones de las palabras claves anteriormente dichas. Después se definió las bases de datos donde se hizo las búsquedas: SCIELO, REDALYC, RESEARCHGATE y repositorios de universidades de Perú, México, Colombia, Ecuador, España, USA, entro otros. Como criterio de exclusión se definió que los artículos que no estaban en los años establecidos (2017 - 2021), por ende, serían sacados debido a que no incluía información actualizada ni uso de nuevas medidas modernas, también se excluyó por estar incompletos, duplicados y por el idioma, enfocándonos en español, inglés y portugués.

\section{RESULTADOS}

La búsqueda de artículos en las bases de datos SCIELO, REDALYC, RESEARCHGATE y repositorios de universidades de Perú, México, Colombia, Ecuador, España, y USA, arrojaron un valor de 48 referencias originales, de las cuales se escogieron 18 entre artículos científicos y proyectos de aplicación para los periodos de 2017 - 2021. A partir de este número se procedió a la investigación de resultados exactos luego de la implementación de tecnologías biométricas en los institutos de educación superior (en especial, las universidades). Dichas entidades reflejaban la tendencia a la aplicación de biometría para el control del acceso por parte de personal y estudiantes a las instituciones. En el análisis de la bibliografía se muestra que, al implementar las tecnologías biométricas en las universidades, se encontró que existen 2 grandes grupos de interés por parte de los investigadores y desarrolladores, las cuales son la biometría dactilar y la biométrica facial aplicables en nuestro campo de interés, que es el control de acceso en las universidades. Después de una revisión sistemática, obtuvimos los siguientes resultados respecto a la implementación de las tecnologías biométricas en el control de acceso en las universidades. Por un lado, en la Tabla 1 abordamos el impacto generado en algunas universidades peruanas y de una forma similar en la Tabla 2, nos centramos en universidades del extranjero, llegando al siguiente resumen:

\begin{tabular}{llllllr}
\hline $\mathbf{N}^{\circ}$ & \multicolumn{1}{c}{ Autor } & \multicolumn{2}{c}{ Título } & Año & País & \multicolumn{2}{c}{ Efectos } \\
\hline $\mathbf{1}$ & José Mercedes & Control de registro de 2017 & Perú & Ahorro de & recursos \\
& Rojas & asistencias para el personal & & & económicos, & horas \\
& Bustamante & administrativo y docente & & empleado y espacio de \\
& & usando relojes biométricos & & & trabajo. & \\
\hline
\end{tabular}


en la universidad nacional

Jorge Basadre.

2 Freddy Roland Implementación del 2017 Perú Satisfacción, seguridad

Garcia Zumaeta Sistema Biométrico para el

Control de Asistencia y veracidad de la

Administrativa de la

Universidad Privada de

Pucallpa 2017

\begin{tabular}{|c|c|c|c|c|c|}
\hline 3 & $\begin{array}{l}\text { Victor Gastelo } \\
\text { Menendez }\end{array}$ & $\begin{array}{l}\text { Propuesta para el diseño de } \\
\text { un sistema de validación y } \\
\text { autentificación biométrico } \\
\text { dactilar para la asociación } \\
\text { guadalupana. }\end{array}$ & 2019 & Perú & $\begin{array}{l}\text { Mejor control de la } \\
\text { seguridad de la } \\
\text { universidad. }\end{array}$ \\
\hline 4 & $\begin{array}{l}\text { Carlos Jhonatan } \\
\text { Llanos } \\
\text { Malpartida }\end{array}$ & $\begin{array}{l}\text { Implementación del lector } \\
\text { biométrico de huella } \\
\text { dactilar para el control de } \\
\text { asistencia de los estudiantes } \\
\text { de la Universidad Nacional } \\
\text { Daniel Alcides Carrión - } \\
\text { Pasco. }\end{array}$ & 2020 & Perú & $\begin{array}{l}\text { Optimización del } \\
\text { tiempo que tomaba } \\
\text { registrar las asistencias. }\end{array}$ \\
\hline
\end{tabular}

Tabla 1: Resumen del efecto de las tecnologías biométricas en la literatura nacional abordada

\begin{tabular}{|c|c|c|c|c|c|}
\hline $\mathbf{N}^{\circ}$ & Autor & Título & Año & País & Efectos \\
\hline 1 & $\begin{array}{l}\text { Jhon Alexander } \\
\text { Calderón }\end{array}$ & $\begin{array}{l}\text { Biometría dactilar: Una } \\
\text { nueva alternativa de } \\
\text { controlar efectivamente la } \\
\text { asistencia a clases }\end{array}$ & 2017 & Colombia & $\begin{array}{l}\text { Disminución de tiempos } \\
\text { en registro, disminución } \\
\text { del uso de papel, }\end{array}$ \\
\hline 2 & $\begin{array}{l}\text { Alma Delia Gonzales } \\
\text { Ramos }\end{array}$ & $\begin{array}{l}\text { Sistema de control de } \\
\text { acceso constituido de } \\
\text { elementos biometricos } \\
\text { (huella dactilar), un }\end{array}$ & 2017 & México & $\begin{array}{l}\text { Ahorro de recursos } \\
\text { económicos, } \\
\text { empleado y espacio de } \\
\text { trabajo. }\end{array}$ \\
\hline
\end{tabular}


microcontrolador (Atmel)

y aplicaciones móviles en

Android

3 Daniel Felipe Sistema de identificación 2017 Colombia Sentirse más seguros en Montaña Duque mediante huella digital un ambiente con mayor para el control de accesos control.

a la universidad libre sede

bosque popular simulado

en un entorno web.

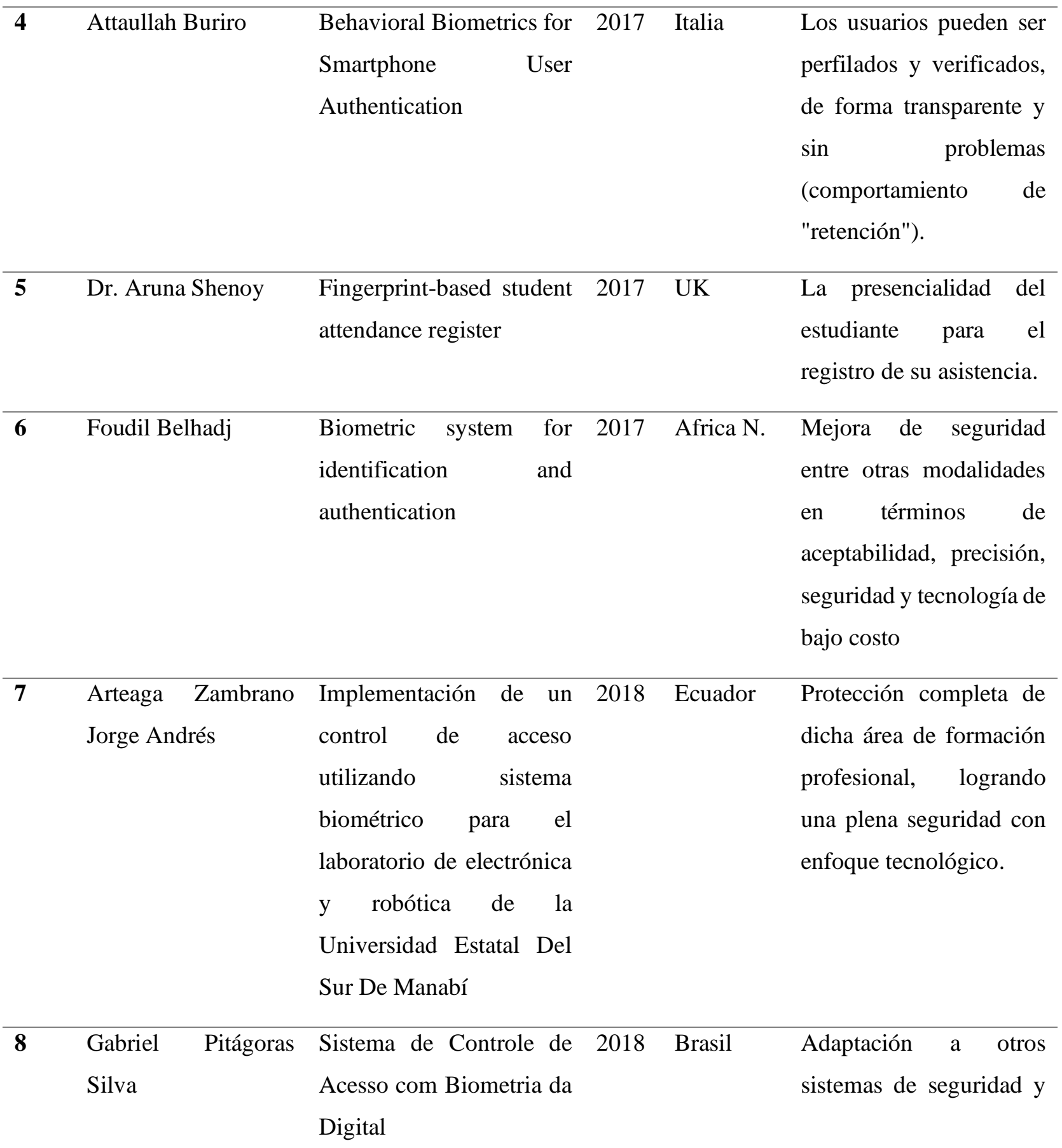


procesos.

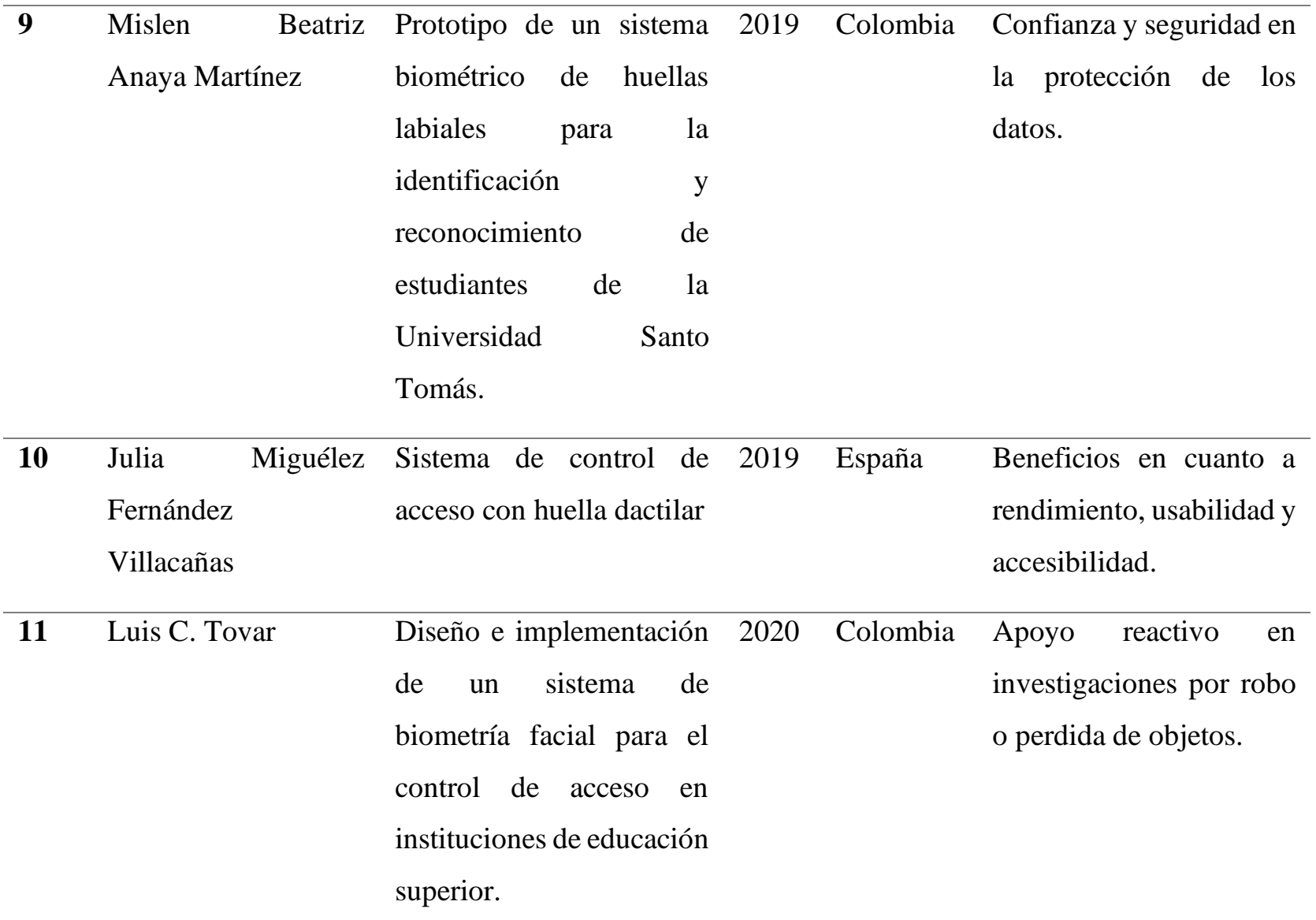

12 Kelvin Agustín Sistema biométrico de 2017 Ecuador Controlar de manera Rosado Cusme control de acceso para el

eficiente el acceso.

laboratorio de cómputo de

la unidad educativa

Francisco González

Álava.

13 Ayang Macdonald Russian university adopts 2021 Rusia Seguridad en campus, STC's facial recognition exámenes a distancia y access control solution presentaciones remotas de trabajos de

calificación.

\begin{tabular}{lll}
\hline Erkam Uzun & Security And Privacy In 2021 USA & \multicolumn{2}{l}{ Los sistemas biométricos } \\
Biometrics-Based & acompañados de \\
Systems & sistemas que le den \\
& soporte, son más eficaces \\
& y seguros.
\end{tabular}


Tabla 2: Resumen del efecto de las tecnologías biométricas en la literatura extranjera abordada

Los datos recolectados en la revisión sistemática, refleja en base a evidencias que todo el conjunto de tecnologías mejoró la actividad respecto a control de asistencia de alumnos, docentes y personal administrativo, evitando los problemas de riesgo de veracidad de la información suministrada por medio de elementos físicos. Si analizamos las diferencias entre la revisión de la bibliografía nacional y la internacional, podemos evidenciar que las tecnologías biométricas más usadas en casi un 100\% de los procesos de control de acceso en universidades peruanas, son las tecnologías biométricas dactilares. Por otro lado, la biometría aplicada en universidades extranjeras es más variada, considerando datos faciales (en su mayoría), labiales, oculares. En la Figura 1, se ha dividido los países de la bibliografía abordada en dos grandes grupos (Biometría dactilar y facial)

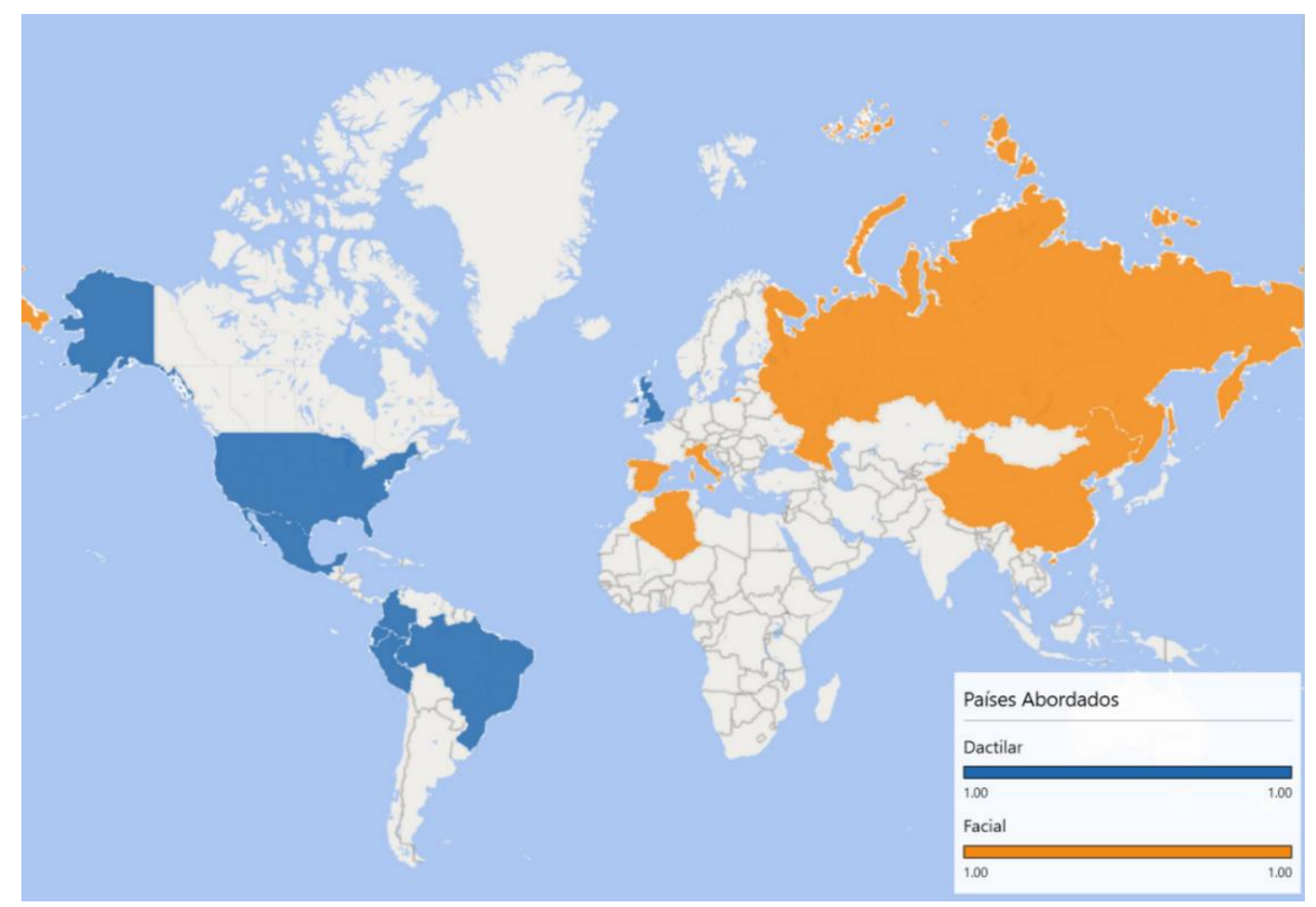

Figura 1. Ubicación geográfica de los países a los que pertenecen las universidades que referencian tendencias pedagógicas de la educación virtual 
El proceso que se planteó en la mayoría de las investigaciones revisadas, fue el uso de la biometría dactilar y facial de la mano de algún sistema. Mediante este se ejecuta la captura de datos de los usuarios (alumno, docente y personal), con la que se almacenan los patrones o características a analizar para gestionar un registro; posterior a eso se realizó un proceso de comparación de las características extraídas por el sistema, y convirtiéndolos en identificadores únicos. Esta salida da como resultado una coincidencia para la posterior toma de decisiones automática, que consiste en aceptar o denegar el marcaje de acceso y asistencia. De acuerdo al análisis y la revisión de la literatura que encierra las diferentes técnicas en las modalidades de control de acceso, se encontró que las tecnologías biométricas de huellas dactilares son las más elegidas en cuanto a beneficio / costo. A su vez, las mismas han estado sufriendo mejoras constantes las cuales ayudan a solventar diversos inconvenientes que se manifiestan a la hora de adquirir la información biométrica de los usuarios, como la suciedad, cicatrices en los dedos y entre otras cosas.

\section{DISCUSIÓN}

Después del proceso de investigación que tiene como objetivo evaluar el impacto que generan las tecnologías biométricas en universidades, podemos ver surgir la siguiente pregunta: ¿Puede la implementación de tecnologías biométricas beneficiar los procesos de control de acceso en las universidades? Al respecto, hay muchos factores que pueden llevarnos a inducir una respuesta positiva, pero naturalmente se debe hacer énfasis en los principales antecedentes, como lo es, por ejemplo, para (Calderon, 2017), el presupuesto que conllevaría la elaboración y mantenimiento de un proyecto de esta escala. Sin embargo, como se analizó en la revisión sistemática, la implementación es claramente viable. Evidentemente, la ejecución e implementación de las tecnologías biométricas en las universidades, también, para el mismo autor, depende de los requerimientos del sistema y de una infraestructura adecuada para disponer de dispositivos necesarios. Es importante precisar en este sentido, que esta variable puede ser mejorada de acuerdo con los dispositivos concretos que se adquieran. De la misma forma lo fundamenta (Anaya Matínez \& Maldonado Arias, 2019), si estas tecnologías cuentan con un nivel de optimización alto, pueden llegar a analizar grandes paquetes de información.

En lo que se refiere a seguridad para, (Garcia \& Hidalgo, 2017), es necesario que la logística sea manejada de la mejor forma y el uso supervisado ya que un dispositivo 
con buen mantenimiento es crucial para lograr un óptimo desempeño en el reconocimiento de datos biométricos. De la misma forma, Tal y como lo especifica (Duque, 2017), las tecnologías biométricas en sistemas, no impide hurtos, pero si ayuda a mitigar el riesgo, es importante seguir construyendo una herramienta que permita asegurar el bienestar tanto de la universidad como de la comunidad estudiantil. Estos programas de última generación brindan confianza y a la vez seguridad en la protección de los datos ya que al estar comprimida en información digital esta puede ser protegida de alteraciones premeditadas y/o accidentales (Parvinzamir, 2017). Aunque los sistemas basados en biometría ofrecen una usabilidad superior y una ventaja para varios casos de uso, tienen que defenderse contra diferentes tipos de ataques de suplantación de identidad y proteger la privacidad de los datos biométricos contra adversarios (Uzun, 2021).

Los factores externos que influyen en las tecnologías biométricas son muy importantes, tal como lo expresa (Gastelo, 2019), referente al personal y usuarios, el autor recomienda la capacitación de ambos elementos para darles a conocer la importancia de la aplicación del proyecto como también su utilización. Los sistemas al estar relacionados con hardware y grandes cantidades de información, se considera necesaria en algunos casos, la utilización de herramientas de cómputo más potentes en cuanto a velocidad y capacidad de procesamiento.

\section{CONCLUSIONES}

Finalizada la revisión sistemática, se pudo identificar los efectos positivos que se obtienen a partir de la aplicación de tecnologías biométricas para el control de acceso en las universidades del mundo, de los cuales se ha obtenido lo siguientes objetos en específico:

- Los sistemas para la validación de las identidades en las universidades son más beneficiosos que el uso de elementos tradicionales con respecto al control de acceso, sin embargo, su implementación y mantenimiento conlleva un aumento en el presupuesto a corto plazo, pero un incremento en la productividad y optimización de procesos a largo plazo.

- La biometría en el control de acceso de estudiantes, docentes y personal brinda seguridad tanto de los datos como del entorno, así como también un manejo más eficiente de los datos. Lo que se evidencia en disminución de tiempos de registros, 
la disminución del uso de documentación excesiva y la centralización de los datos de forma óptima y segura.

- Las tecnologías biométricas utilizan atributos identificables únicos de las personas para la identificación y autenticación. Lo que conlleva a la agilización en el control de acceso, reduciendo tiempos de espera, generando así un incremento en la satisfacción de usuarios en general.

- Las tecnologías biométricas más usadas para el control de acceso a las universidades, según la presente investigación, son la biometría dactilar y la biometría facial. Por lo mismo, estas se pueden utilizar en casi cualquier aplicación que requiera la identificación exacta de un individuo. Esto va desde computadoras donde el escaneo de la huella dactilar para verificar la identidad de un usuario de manera simple, hasta la implementación de escáneres para el reconocimiento de patrones y rasgos únicos de cada individuo.

\section{REFERENCIAS BIBLIOGRÁFICAS}

Anaya Matínez, M. B., \& Maldonado Arias, D. S. (2019). Prototipo de un sistema biométrico de huellas labiales para la identificación y reconocimiento de estudiantes de la Universidad Santo Tomás.

Arteaga Zambrano, A. (2018). IMPLEMENTACIÓN DE UN CONTROL DE ACCESO UTILIZANDO SISTEMA BIOMÉTRICO PARA EL LABORATORIO DE ELECTRÓNICA Y ROBÓTICA DE LA UNIVERSIDAD ESTATAL DEL SUR DE MANABÍ. 106.

Belhadj, F. (2017). Biometric system for identification and authentication. 117.

Buriro, A. (2017). Behavioral Biometrics for Smartphone User Authentication [Phd, University of Trento]. http://eprints-phd.biblio.unitn.it/1935/

Bustamante, R., \& Mercedes, J. (s. f.). CONTROL DE REGISTRO DE ASISTENCIA PARA EL PERSONAL ADMINISTRATIVO Y DOCENTE UTILIZANDO RELOJES BIOMÉTRICOS Y RADIOENLACES EN LA UNIVERSIDAD NACIONAL JORGE BASADRE GROHMANN TACNA. 103.

Calderon, J. A. (2017). Biometría dactilar: Una nueva alternativa de controlar efectivamente la asistencia a clases | Investigación e Innovación en Ingenierías. Universidad Simón Bolívar. https://doi.org/10.17081/invinno.6.1.2773 
Delia, A. (2017). Sistema de control de acceso constituido de elementos biometricos (huella dactilar), un microcontrolador (Atmel) y aplicaciones móviles en Android. 6.

Duque, D. F. M. (2017). SISTEMA DE IDENTIFICACIÓN MEDIANTE HUELLA DIGITAL PARA EL CONTROL DE ACCESOS A LA UNIVERSIDAD LIBRE SEDE BOSQUE POPULAR SIMULADO EN UN ENTORNO WEB. 80.

Fernández Villacañas, J. M., Navazo Escudero, A., Fernández Villacañas, J. M., \& Navazo Escudero, A. (2019, septiembre 20). Sistema de control de acceso con huella $\begin{array}{llll}\text { dactilar } & y & \text { Arduino } & \text { [Info:eu-repo/semantics/bachelorThesis]. }\end{array}$ https://eprints.ucm.es/id/eprint/65221/

Garcia, F. R., \& Hidalgo, H. (2017). Implementación del Sistema Biométrico para el Control de Asistencia Administrativa de la Universidad Privada de Pucallpa S.A.C 2017.

Gastelo, V. (2019). Propuesta para el diseño de un sistema de validación y autentificación biométrico dactilar para la asociación guadalupana.

LLANOS MALPARTIDA, C. J. (2020). Implementación del lector biométrico de huella dactilar para el control de asistencia de los estudiantes de la Universidad Nacional Daniel Alcides Carrión - Pasco. 99.

Macdonald, A. (2021). Russian university adopts STC's facial recognition access control solution | Biometric Update. https://www.biometricupdate.com/202102/russianuniversity-adopts-stcs-facial-recognition-access-control-solution

Parvinzamir, F. (2017). Fingerprint-based Student Attendance Register. 91.

Peralta Castellano, J. C., \& Peralta Castellano, J. C. (2015). Nueve años de biometría en el Perú: La fe de identificación en la encrucijada. Revista IUS, 9(36), 275-301.

Silva, G. P. (2018). Sistema de Controle de Acesso com Biometria da Digital. VIII Simposio de Excelencia em Gestao e Tecnologia.

Tovar, L. C., Echavez, M. E., Universidad de Cartagena, Colombia, Martelo, R. J., \& Universidad de Cartagena, Colombia. (2020). Diseño e implementación de un sistema de biometría facial para el control de acceso en instituciones de educación superior. Espacios, 41(44). https://doi.org/10.48082/espacios-a20v41n44p26 
Uzun, E. (2021). A Dissertation Presented to The Academic Faculty. SECURITY AND PRIVACY IN BIOMETRICS, 162.

Zhou, X. (2020). Application Research of Face Recognition Technology in Smart Campus. Journal of Physics: Conference Series, 1437(1), 012130. https://doi.org/10.1088/1742-6596/1437/1/012130 